\title{
Quantitative grain-scale ferroic domain volume fractions and domain switching strains from three-dimensional X-ray diffraction data
}

Oddershede, Jette; Majkut, Marta; Caosyd, Qinghua ; Schmidt, Søren; Wright, Jonathan P.; Kenesei, Peter; Daniels, John E.

Published in:

Journal of Applied Crystallography

Link to article, DOI:

$10.1107 / \mathrm{S} 1600576715007669$

Publication date:

2015

Document Version

Publisher's PDF, also known as Version of record

Link back to DTU Orbit

Citation (APA):

Oddershede, J., Majkut, M., Caosyd, Q., Schmidt, S., Wright, J. P., Kenesei, P., \& Daniels, J. E. (2015).

Quantitative grain-scale ferroic domain volume fractions and domain switching strains from three-dimensional $X$ ray diffraction data. Journal of Applied Crystallography, 48, 882-889.

https://doi.org/10.1107/S1600576715007669

\section{General rights}

Copyright and moral rights for the publications made accessible in the public portal are retained by the authors and/or other copyright owners and it is a condition of accessing publications that users recognise and abide by the legal requirements associated with these rights.

- Users may download and print one copy of any publication from the public portal for the purpose of private study or research.

- You may not further distribute the material or use it for any profit-making activity or commercial gain

- You may freely distribute the URL identifying the publication in the public portal 


\section{Quantitative grain-scale ferroic domain volume fractions and domain switching strains from three-dimensional X-ray diffraction data}

Jette Oddershede, Marta Majkut, Qinghua Cao, Søren Schmidt, Jonathan P. Wright, Peter Kenesei and John E. Daniels

J. Appl. Cryst. (2015). 48, 882-889

\section{IUCr Journals CRYSTALLOGRAPHY JOURNALS ONLINE}

Copyright (C) International Union of Crystallography

Author(s) of this paper may load this reprint on their own web site or institutional repository provided that this cover page is retained. Republication of this article or its storage in electronic databases other than as specified above is not permitted without prior permission in writing from the IUCr.

For further information see http://journals.iucr.org/services/authorrights.html 
JOURNAL OF

APPLIED

CRYSTALLOGRAPHY

ISSN 1600-5767

Received 3 February 2015

Accepted 18 April 2015

Edited by D. Pandey, Indian Institute of Technology (Banaras Hindu University), Varanasi, India

Keywords: ferroic materials; domain volume fractions; grain-scale strain; three-dimensional X-ray diffraction.

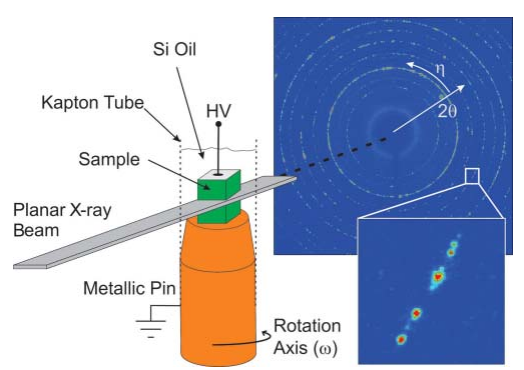

(C) 2015 International Union of Crystallography

\section{Quantitative grain-scale ferroic domain volume fractions and domain switching strains from three- dimensional $\mathrm{X}$-ray diffraction data}

\author{
Jette Oddershede, ${ }^{\mathrm{a} *}$ Marta Majkut, ${ }^{\mathrm{a}}$ Qinghua Cao, ${ }^{\mathrm{b}}$ Søren Schmidt, ${ }^{\mathrm{a}}$ Jonathan P. \\ Wright, ${ }^{\mathrm{c}}$ Peter Kenesei ${ }^{\mathrm{d}}$ and John E. Daniels ${ }^{\mathrm{b}}$
}

${ }^{a}$ NEXMAP, DTU Physics, 2800 Kongens Lyngby, Denmark, ${ }^{\mathbf{b}}$ School of Materials Science and Engineering, UNSW Australia, Sydney, NSW 2052, Australia, 'European Synchrotron Radiation Facility, Grenoble, BP-220, France, and ${ }^{\mathbf{d}} 1$ ID, Advanced Photon Source, Lemont, IL 60439, USA. *Correspondence e-mail: jeto@fysik.dtu.dk

A method for the extension of the three-dimensional X-ray diffraction technique to allow the extraction of domain volume fractions in polycrystalline ferroic materials is presented. This method gives access to quantitative domain volume fractions of hundreds of independent embedded grains within a bulk sample. Such information is critical to furthering our understanding of the grainscale interactions of ferroic domains and their influence on bulk properties. The method also provides a validation tool for mesoscopic ferroic domain modelling efforts. The mathematical formulations presented here are applied to tetragonal coarse-grained $\mathrm{Ba}_{0.88} \mathrm{Ca}_{0.12} \mathrm{Zr}_{0.06} \mathrm{Ti}_{0.94} \mathrm{O}_{3}$ and rhombohedral fine-grained (0.82) $\mathrm{Bi}_{0.5} \mathrm{Na}_{0.5} \mathrm{TiO}_{3}-(0.18) \mathrm{Bi}_{0.5} \mathrm{~K}_{0.5} \mathrm{TiO}_{3}$ electroceramic materials. The fitted volume fraction information is used to calculate grain-scale non- $180^{\circ}$ ferroelectric domain switching strains. The absolute errors are found to be approximately 0.01 and $0.03 \%$ for the tetragonal and rhombohedral cases, which had maximum theoretical domain switching strains of 0.47 and $0.54 \%$, respectively. Limitations and possible extensions of the technique are discussed.

\section{Introduction}

Bulk polycrystalline ferroic materials find a broad range of practical applications, such as AC transformer cores (ferromagnetics), superelastic constant-force springs (ferroelastics) and high-strain electromechanical actuators (ferroelectrics). All of these materials undergo a phase transformation to the ferroic state upon cooling or under the influence of a field, such as magnetic, electric or stress field. During the phase transformation, ferroic domains are formed within the material. The morphology of the resultant domain structures depends on many factors, including (but by no means limited to) the magnitude and crystallographic orientation of the ferroic order parameter, the polycrystalline microstructure, and the anisotropy of the driving field. This morphology is one of the key structural parameters that influence the bulk properties of ferroic materials, as these properties are governed by the movement of domain boundaries and their interactions with one another and with other microstructural defects. Furthermore, the discovery of functionality of domain walls (Seidel et al., 2009) has opened new possibilities with regards to potential ferroic material properties. Characterization of domain structures in the bulk of polycrystalline ferroic materials is therefore critical to further understand and engineer properties of such materials. This paper concentrates on the measurement of non- $180^{\circ}$ ferroelectric domains, which possess a spontaneous strain; however, as discussed in $\S 5$, the 
technique may be extended and applied in many other materials.

In the case of ferroelectric materials, domain information can be observed using a range of methods. A recent review article by $\mathrm{Wu}$ et al. (2015) provides an excellent overview. Optical microscopy, either with preferential etching of domain orientations or using polarized light, can distinguish certain types of domain structures intersecting the surface of a material. These surface images have been used to infer the three-dimensional domain structures in the bulk (Arlt \& Sasko, 1980). Likewise, scanning probe techniques can also detect domain structures at the surface but have the additional advantage of being able to resolve ferroelectric polarization vectors (Gruverman et al., 1995). Transmission electron microscopy can be used to observe domain structures at very small length scales and under in situ electric fields (Tan et al., 2005) but is only applicable to very small sample sizes. A digital holography technique has been demonstrated to detect domain structures in three dimensions where volume-fraction information is also accessible (Zhi et al., 2009); however, in this case the method is somewhat limited to single crystals with certain optical properties.

Another approach to gain information on domain processes in the bulk is to measure average domain populations amongst grain families using powder X-ray or neutron diffraction (Lupascu et al., 2001; Hall et al., 2004; Pramanick et al., 2011). These methods have significantly advanced our understanding of the effect of non- $180^{\circ}$ ferroelectric domain wall motion on the properties of bulk electroceramics by allowing for in situ measurement of the rearrangement of domains under electric field and stress. Additionally, these methods have allowed for the characterization of general grain-orientation-dependent strains associated with non- $180^{\circ}$ ferroelectric domain wall motion and have been applied both statically and dynamically under an applied electric field (Wang et al., 2014) and stress (Harrison, 2004).

None of these methods, however, provide access to volume fraction information from isolated grains within bulk polycrystals, leaving remaining questions as to the role of local grain neighbourhoods in determining the bulk properties of ferroic polycrystals.

Here we demonstrate the collection and analysis of threedimensional X-ray diffraction (3D-XRD) data to obtain the domain volume fractions resolved at the grain scale from within a bulk electroceramic. While anomalous scattering methods have been used to resolve mechanisms of $180^{\circ}$ ferroelectric domain switching (Azimonte et al., 2010), the present method is only sensitive to non- $180^{\circ}$ ferroelectric domain volume fraction changes. These changes give rise to an induced non- $180^{\circ}$ ferroelectric domain switching strain, the magnitude of which is calculated here for both tetragonal and rhombohedral symmetries. The methods demonstrated are by no means limited to the case of electroceramics. They can be applied to all ferroic materials that possess a spontaneous strain, which results in the separation of scattered intensity from domain components in diffraction space. Possible extensions to the data collection methods can be envisioned where the requirement for a spontaneous strain need not be fulfilled. For example, using neutrons with polarization analysis would allow for the assignment of magnetic domain orientations within ferromagnetic materials. Likewise, resolving all ferroelectric domains without a coupled spontaneous strain could potentially be achieved using anomalous or polarized X-ray scattering.

\section{Experimental details}

Details of the materials processing for the specific compositions used are provided in subsequent sections. Samples of suitable geometry were cut from bulk polycrystalline ceramics in a rectangular shape. Gold electrodes were sputtered onto two opposing surfaces. The samples were then placed with an electrode surface in contact with a brass pin and electrically contacted using silver paint. A top electrode wire was connected using silver paint and the sample encapsulated in a $1 \mathrm{~mm}$-diameter Kapton tube filled with silicone oil. Such a setup allows the application of high electric fields without the risk of dielectric breakdown around the sample edges (Daniels et al., 2009).

The 3D-XRD method allows for the indexing of many individual grains from a bulk material and gives direct access to grain-resolved information on position, orientation, volume and strain (Poulsen et al., 2001; Poulsen, 2004; Juul Jensen et al., 2006; Oddershede et al., 2010; Bernier et al., 2011; Sørensen et al., 2012). A schematic diagram of the 3D-XRD setup that allows for in situ electrical loading and resulting diffraction patterns is shown in Fig. 1. The method requires that the X-ray beam illuminates a limited number of grains at a given time. The total number of grains is a function of (1) the beam size, (2) the sample grain size and (3) the sample thickness. Thus, these parameters need to be optimized in order to obtain scattering patterns appropriate for analysis. To probe a large fraction of reciprocal space for each grain, the sample is rotated around the common rotation and poling axis $\omega$ in steps

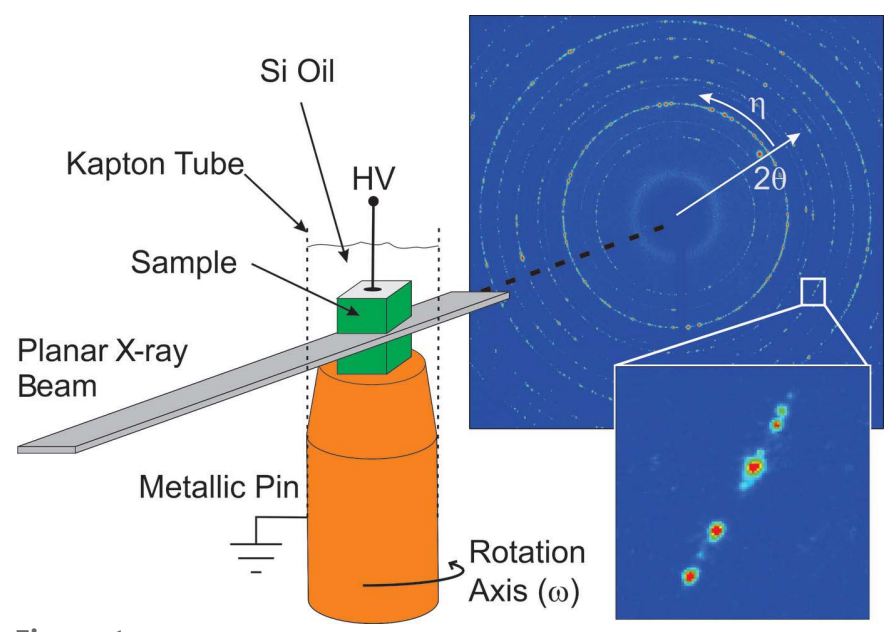

Figure 1

Schematic diagram of the experimental setup. A planar beam intersects the sample giving rise to 'spotty' diffraction rings owing to the limited number of grains sampled. A single image is obtained for each rotational increment $\Delta \omega$. 
of $\Delta \omega$. In the ideal setup, the beam width is larger than the sample cross section such that the illuminated volume contains the same grains throughout the full rotation of the sample. However, many ferroelectric materials of interest have grain sizes less than $5 \mu \mathrm{m}$, limiting the maximum sample cross section for full illumination to approximately $100 \mu \mathrm{m}$ or less. Such samples can be difficult to produce, so it is often necessary to reduce the beam width in order to limit the number of overlapping diffraction spots.

The collected sequences of diffraction images were analysed using the Fable software package (http://sourceforge. net/p/fable/wiki/Home/). The first step in the analysis procedure is to identify the coordinates $(2 \theta, \eta, \omega)$ of all observed diffraction spots, where $2 \theta$ refers to the scattering angle (radial direction on the detector), $\eta$ the azimuthal position on the detector and $\omega$ the rotation angle of the sample (see Fig. 1 for reference). In the subsequent indexing step, performed using GrainSpotter (Schmidt, 2014), the diffraction spots are assigned to individual grains, and the orientations and positions of these grains are refined. The version of GrainSpotter used for the present study only considers grains with a high completeness in terms of the ratio of the number of assigned to expected reflections within specified tolerances in $2 \theta, \eta$ and $\omega$. It does not allow grains to share reflections, and since many reflections are in fact shared between ferroelastic domains, this in practice means that in most cases only one domain from each grain will be indexed. The results from the indexing include a list of grain orientations, positions and corresponding reflections. These, along with the collected sequence of diffraction images, comprise the input for the domain volume fitting algorithm.

\section{Algorithm details}

\subsection{Resolving ferroelastic domain volume fractions}

Individual peak families from a given grain are extracted from the data volume ( $h k l$ indices refer to the primitive cubic unit cell with $a \simeq 4 \AA$ ). For the three non- $180^{\circ}$ tetragonal domains $d_{200}, d_{020}$ and $d_{002}$ the $\{002\}$ reflections carry information about the domain volume fractions $v_{200}, v_{020}$ and $v_{002}$, whereas the $\{111\}$ reflections can be used to extract domain volume fractions $v_{\overline{1} 11}, v_{1 \overline{1} 1}, v_{11 \overline{1}}$ and $v_{111}$ for the four non- $180^{\circ}$ rhombohedral domains $d_{\overline{1} 11}, d_{1 \overline{1} 1}, d_{11 \overline{1}}$ and $d_{111}$. Each reflection is then integrated into a radial profile along $2 \theta$, and the resulting peaks are fitted using two Gaussian peak functions centred at $2 \theta_{\min }$ and $2 \theta_{\max }$. The intensities $I\left(2 \theta_{\min }\right)$ and $I\left(2 \theta_{\max }\right)$ of the two Gaussian peaks are then corrected for the Lorentz and polarization factors. Thereafter, they are proportional to the sum of domain volumes from particular domain variants. For instance, in the case of rhombohedral symmetry, the volume fraction $v_{11 \overline{1}}$ of domain $d_{11 \overline{1}}$ must equal the ratio of $I^{11 \overline{1}}\left(2 \theta_{\min }\right)$ to the total intensity $I_{\text {tot }}^{11 \overline{1}}=I^{11 \overline{1}}\left(2 \theta_{\min }\right)+I^{11 \overline{1}}\left(2 \theta_{\max }\right)$ for each of the $11 \overline{1}$ and $\overline{11} 1$ reflections. This assumes that all variants of the peak family have the same scattering factors, which is considered a reasonable assumption given that the distortion away from cubic is less than $1 \%$ for the sample materials in this study (see $\$ 4$ ).
3D-XRD experiments with in situ electrical loading typically cover an $\omega$ rotation range of $320-340^{\circ}$ because the support for the top electrode connection shadows part of the incoming and diffracted beams. Thus, most reflections are measured twice (on the left and right side of the detector). Including Friedel pairs, this gives up to four observations for each domain volume fraction. This enables us to set up a leastsquares fit of the domain volume fractions for individual grains. For tetragonal symmetry the residual $\chi^{2}$ that we need to minimize is defined as

$$
\chi_{\mathrm{tetr}}^{2}=\sum_{t=(200,020,002)} \sum_{i=1}^{n(t)} \frac{1}{w_{t, i}}\left[v_{t}-\frac{I^{t, i}\left(2 \theta_{\min }\right)}{I_{\mathrm{tot}}^{t, i}}\right]^{2},
$$

whereas for rhombohedral symmetry we have

$$
\chi_{\mathrm{rh}}^{2}=\sum_{t=(\overline{1} 11,1 \overline{1} 1,11 \overline{1}, 111)} \sum_{i=1}^{n(t)} \frac{1}{w_{t, i}}\left[v_{t}-\frac{I^{t, i}\left(2 \theta_{\mathrm{min}}\right)}{I_{\mathrm{tot}}^{t, i}}\right]^{2} .
$$

Here the sum over $i$ runs over the number of observations $[0 \leq$ $n(t) \leq 4]$ of each reflection type $t$. In order to constrain the sum of volume fractions to unity we replace $v_{002}$ by $1-v_{200}-v_{020}$ in $\chi_{\text {tetr }}^{2}$, and $v_{111}$ by $1-v_{\overline{1} 11}-v_{1 \overline{1} 1}-v_{11 \overline{1}}$ in $\chi_{\mathrm{rh}}^{2}$. The weights $w_{t, i}$ can be set to weight all observed reflections equally $\left(w_{t, i}=1\right)$, to compensate for the different number of observations of each type $\left[w_{t, i}=n(t)\right]$ or to down-weight outlier intensities, caused for example by overlapping reflections from different grains or by the grain of interest rotating partially out of the illuminated volume if the beam width is smaller than the sample cross section. This can for instance be done by using as $w_{t, i}$ the absolute deviation of $I_{\mathrm{tot}}^{t, i}$ either from the median over all reflections assigned to the grain or from the median over the type $t$ subset of these reflections.

It is possible to minimize equation (1) if reflections corresponding to two of the three possible tetragonal domains are measured, and likewise for equation (2) with three of the four rhombohedral domains represented. This allows a result to be obtained even when given reflections are missing from the data set owing to incomplete sampling of reciprocal space. The actual minimization of the residuals is performed by means of the Levenberg-Marquardt algorithm (Levenberg, 1944; Marquardt, 1963). The output is a vector containing the domain volume fractions, either $\mathbf{v}_{\text {tetr }}=\left(v_{200}, v_{020}, 1-v_{200}-\right.$ $\left.v_{020}\right)$ or $\mathbf{v}_{\mathrm{rh}}=\left(v_{\overline{1} 11}, v_{1 \overline{1} 1}, v_{11 \overline{1}}, 1-v_{\overline{1} 11}-v_{1 \overline{1} 1}-v_{11 \overline{1}}\right)$, and the corresponding covariance matrix, $\Sigma_{\text {tetr }}$ or $\Sigma_{\mathrm{rh}}$, respectively.

\subsection{Calculating strain along the electric field vector}

The strain along the electric field vector resulting from non$180^{\circ}$ ferroelectric domain switching can be calculated once the domain volume fractions have been obtained as outlined by Hall and co-workers for tetragonal (Hall et al., 2005) and later also for rhombohedral (Hall et al., 2006) symmetry. The error estimate on a domain switching strain can be propagated from the covariances. In the following we let $\mathbf{I}=\left(l_{1}, l_{2}, l_{3}\right)$ be a unit vector along the poling direction in the crystal reference frame, and reproduce the definitions of the poling strain along $\mathbf{l}, \varepsilon_{\mathrm{p}}$. 
3.2.1. Tetragonal symmetry. For tetragonal symmetry the poling strain along $\mathbf{I}$ is defined as (Hall et al., 2005)

$$
\begin{aligned}
\varepsilon_{\mathrm{p}} & =\left(\frac{c-a}{a_{0}}\right)\left(v_{200} l_{1}^{2}+v_{020} l_{2}^{2}+v_{002} l_{3}^{2}-\frac{1}{3}\right) \\
& =\left(\frac{c-a}{a_{0}}\right)\left[\left(v_{200}, v_{020}, v_{002}\right) \cdot \mathbf{L}_{\mathrm{tetr}}-\frac{1}{3}\right],
\end{aligned}
$$

where $c$ and $a$ are the tetragonal lattice parameters, and $a_{0}$ is the lattice parameter of the cubic phase. Without an independent measure of the cubic lattice parameter one can use $a_{0}=\left(c a^{2}\right)^{1 / 3}$, thus assuming no volume change at the nonferroic to ferroic phase transition. For $\mathbf{I}=\langle 111\rangle$ or $v_{200}=$ $v_{020}=v_{002}=1 / 3$ we get $\varepsilon_{\mathrm{p}}=0$ because $v_{200}+v_{020}+v_{002}=1$ and $l_{1}^{2}+l_{2}^{2}+l_{3}^{2}=1$. The maximum poling strain of $2(c-a) /\left(3 a_{0}\right)$ is obtained for a single domain with $\langle 100\rangle$ aligned along the electric field direction, while the minimum possible value of $\varepsilon_{\mathrm{p}}$ is $(a-c) /\left(3 a_{0}\right)$. The error estimate on the poling strain can then be propagated as

$$
\begin{aligned}
\sigma\left(\varepsilon_{\mathrm{p}}\right) & =\left|\frac{c-a}{a_{0}}\right|\left[\left(l_{1}^{2}, l_{2}^{2}, l_{3}^{2}\right) \Sigma_{\text {tetr }}\left(l_{1}^{2}, l_{2}^{2}, l_{3}^{2}\right)^{\mathrm{T}}\right]^{1 / 2} \\
& =\left|\frac{c-a}{a_{0}}\right|\left(\mathbf{L}_{\text {tetr }} \Sigma_{\text {tetr }} \mathbf{L}_{\text {tetr }}^{\mathrm{T}}\right)^{1 / 2},
\end{aligned}
$$

assuming that the experimental error on the orientation of the electric field direction is negligible.

3.2.2. Rhombohedral symmetry. For rhombohedral symmetry the poling strain along $\mathbf{I}$ is defined as (Hall et al., 2006)

$$
\begin{aligned}
\varepsilon_{\mathrm{p}}= & 2 \gamma\left[v_{\overline{1} 11}\left(-l_{1} l_{2}-l_{1} l_{3}+l_{2} l_{3}\right)+v_{1 \overline{1}}\left(-l_{1} l_{2}+l_{1} l_{3}-l_{2} l_{3}\right)\right. \\
& \left.+v_{11 \overline{1}}\left(l_{1} l_{2}-l_{1} l_{3}-l_{2} l_{3}\right)+v_{111}\left(l_{1} l_{2}+l_{1} l_{3}+l_{2} l_{3}\right)\right] \\
= & 2 \gamma\left(v_{\overline{1} 11}, v_{1 \overline{1} 1}, v_{11 \overline{1}}, v_{111}\right) \cdot \mathbf{L}_{\mathrm{rh}},
\end{aligned}
$$

where $2 \gamma=\pi\left(1 / 2-\alpha_{\mathrm{rh}} / 180^{\circ}\right)$ is the maximum poling strain, obtained for a single domain with a $\langle 111\rangle$ axis aligned along the electrical field direction. For $\mathbf{I}=\langle 100\rangle$ or $v_{\overline{1} 11}=v_{1 \overline{1} 1}=$ $v_{11 \overline{1}}=v_{111}=1 / 4$ we get $\varepsilon_{\mathrm{p}}=0$, while the minimum value of $\varepsilon_{\mathrm{p}}$ is $-\gamma$. The error estimated on the poling strain is then given as

$$
\sigma\left(\varepsilon_{\mathrm{p}}\right)=2 \gamma\left(\mathbf{L}_{\mathrm{rh}} \Sigma_{\mathrm{rh}} \mathbf{L}_{\mathrm{rh}}^{\mathrm{T}}\right)^{1 / 2}
$$

where $\mathbf{L}_{\mathrm{rh}}$ is defined in equation (5) and again the experimental error on the electric field direction is ignored.

\section{Applications}

The maximum poling strain possible for a given crystal symmetry and grain orientation is obtained when the grain texture is saturated, meaning that the entire grain is transformed to the domain variant with a polarization axis (and corresponding spontaneous strain) most favourably oriented relative to the applied field direction. The maximum poling strain is therefore also termed the theoretical saturated strain. Fig. 2 shows a simulation of theoretical saturated strains for 10000 randomly oriented cubic grains that are assumed to transform to either tetragonal or rhombohedral symmetry. The tetragonal and rhombohedral lattice parameters are taken

to be the same as in the two experiments described below on samples of the same respective crystallographic symmetries.

\subsection{Material 1: tetragonal coarse-grained BCZT}

4.1.1. Sample preparation. Samples of $\mathrm{Ba}_{0.88} \mathrm{Ca}_{0.12} \mathrm{Zr}_{0.06}$ $\mathrm{Ti}_{0.94} \mathrm{O}_{3}$ (BCZT) were produced by the mixed oxide method. Details of this material system and the resulting electromechanical properties can be found elsewhere (Liu \& Ren, 2009; Li et al., 2010). The resulting grain size of the samples was approximately $20-40 \mu \mathrm{m}$. A sample was cut and polished into a cuboid shape of dimensions $300 \times 300 \times 300 \mu \mathrm{m}$ and prepared for in situ measurements as outlined in $\S 2$.

4.1.2. Data acquisition. X-ray diffraction experiments were performed at beamline 1-ID-E of the Advanced Photon Source, Argonne National Laboratory. A beam energy of $61.332 \mathrm{keV}$ ( $\mathrm{Yb} K$ edge) was focused and trimmed to a planar beam of dimensions $600 \mu \mathrm{m}$ in width $\times 30 \mu \mathrm{m}$ in height at the sample position, thus illuminating the entire sample cross section. The sample was then rotated around $\omega$ while data were collected over $2 \times 166^{\circ}$ in $0.1^{\circ}$ integration angles with an exposure time of $0.2 \mathrm{~s}$ per image. A GE 41RT detector with $2048 \times 2048$ pixels of $200 \times 200 \mu \mathrm{m}$ was employed at a sample-to-detector distance of $1560 \mathrm{~mm}$. Four $30 \mu \mathrm{m}$-thick layers perpendicular to the common electric field and rotation axis were collected with an interlayer spacing of $15 \mu \mathrm{m}$, both in the initial unpoled state and in the remnant state, after the sample had been electrically poled with a field of $1.5 \mathrm{kV} \mathrm{mm}^{-1}$.

4.1.3. Grain indexing and selection. From the powder diffraction pattern of the same sample the tetragonal lattice parameters of the BCZT composition were refined to $a=$ $3.993 \AA$ and $c=4.021 \AA$. GrainSpotter (Schmidt, 2014) was then used to index 150-160 tetragonal domains in every illuminated layer of the sample. A matching procedure was performed to identify exactly one domain orientation for each grain and to track this orientation from one layer to the next. Because the average grain size is larger than the beam height, reflection intensities corresponding to each grain across the four illuminated layers were then compared to exclude partly illuminated grains with maximum intensities in the top or bottom layers. This leaves 105 grains centrally located in the volume of interest for further analysis.

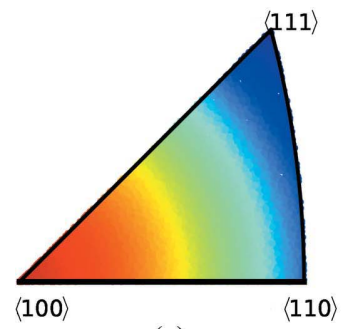

(a)

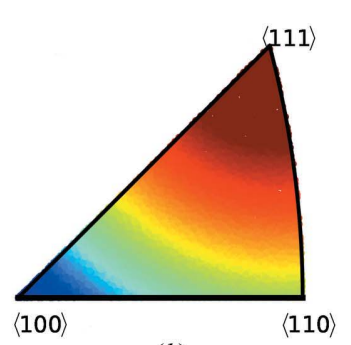

(b)

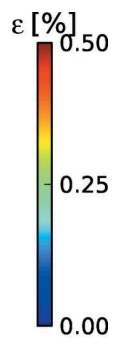

Figure 2

Theoretical saturated domain texture strains for 10000 randomly oriented grains with ( $a$ ) tetragonal symmetry, $a=3.993 \AA, c=4.021 \AA$ and $(b)$ rhombohedral symmetry, $\alpha_{\mathrm{rh}}=89.69^{\circ}$. For these conditions the maximum poling strain is $\varepsilon_{\mathrm{p}}^{\text {sat }}=0.466 \%$ and $\varepsilon_{\mathrm{p}}^{\text {sat }}=0.547 \%$ for the tetragonal and rhombohedral cases, respectively. 
Table 1

Details of four selected grains for which the domain volume fraction algorithm has been employed.

$T_{100}$ and $T_{111}$ are from coarse-grained tetragonal BCZT ( $\left.\$ 4.1\right)$, while $R_{100}$ and $R_{111}$ are from fine-grained rhombohedral BNKT ( $\left.\$ 4.2\right)$.

\begin{tabular}{lllllllll}
\hline & $\begin{array}{l}\varepsilon_{\mathrm{p}} \\
(\%)\end{array}$ & $\begin{array}{l}\varepsilon_{\mathrm{p}}^{\text {sat }} \\
(\%)\end{array}$ & $\begin{array}{l}\alpha_{200} \\
\left({ }^{\circ}\right)\end{array}$ & $\begin{array}{l}\alpha_{020} \\
\left({ }^{\circ}\right)\end{array}$ & $\begin{array}{l}\alpha_{002} \\
\left({ }^{\circ}\right)\end{array}$ & $v_{200}$ & $v_{020}$ & \multicolumn{1}{c}{$v_{002}$} \\
\hline$T_{100}$ & $0.064(3)$ & 0.46 & 84 & 89 & 7 & $0.203(4)$ & $0.370(4)$ & $0.427(5)$ \\
$T_{111}$ & $0.0035(2)$ & 0.03 & 58 & 54 & 52 & $0.322(4)$ & $0.224(3)$ & $0.453(4)$ \\
\hline
\end{tabular}

\begin{tabular}{|c|c|c|c|c|c|c|c|c|c|c|}
\hline & $\begin{array}{l}\varepsilon_{\mathrm{p}} \\
(\%)\end{array}$ & $\begin{array}{l}\varepsilon_{\mathrm{p}}^{\text {sat }} \\
(\%)\end{array}$ & $\begin{array}{l}\alpha_{\overline{1} 11} \\
\left({ }^{\circ}\right)\end{array}$ & $\begin{array}{l}\alpha_{1 \overline{1} 1} \\
\left({ }^{\circ}\right)\end{array}$ & $\begin{array}{l}\alpha_{11 \overline{1}} \\
\left({ }^{\circ}\right)\end{array}$ & $\begin{array}{l}\alpha_{111} \\
\left({ }^{\circ}\right)\end{array}$ & $v_{\overline{1} 11}$ & $v_{1 \overline{1} 1}$ & $v_{11 \overline{1}}$ & $v_{111}$ \\
\hline$R_{100}$ & $-0.07(1)$ & 0.21 & 70 & 40 & 58 & 54 & $0.41(2)$ & $0.07(2)$ & $0.30(5)$ & $0.22(5)$ \\
\hline$R_{111}$ & $0.28(2)$ & 0.52 & 64 & 67 & 81 & 11 & $0.23(3)$ & $0.14(2)$ & $0.00(6)$ & $0.63(3)$ \\
\hline
\end{tabular}

To demonstrate the algorithm, two of the 105 grains in the remnant state were selected. These grains have the electric field vector close to the crystallographic [100] $\left(T_{100}\right)$ and [111] $\left(T_{111}\right)$ axes and are thus expected to display high and low poling strains, respectively ( $c f$. Fig. $2 a$ ). The resulting domain volume fractions and poling strains given in Table 1 are calculated as volume-weighted averages over grain slices from non-overlapping layers. Here the volume of a grain slice is calculated as the median over $I_{\text {tot }}^{t, i}$ for all corresponding reflections, while the error bar is estimated from the median absolute deviation of $I_{\text {tot }}^{t, i}$. The example reflections in Fig. 3 are from the layer where the slice volume of grain $T_{100}$ was maximum.

4.1.4. Domain volume fitting. Fig. 3 shows the integrated reflections for grain $T_{100}$. The overall intensities are observed to be very consistent for all 12 measured reflections, and the same holds for the $I\left(2 \theta_{\text {min }}\right): I_{\text {tot }}$ ratios for each domain type.
On this basis this the weights $w_{t, i}$ of equation (1) range from 0.9 to 3.7 , taking into account both the overall intensity consistency and the consistency within the group of reflections corresponding to a certain domain type. The absolute error bars on the resulting domain volume fractions and poling strain in Table 1 are less than $0.01 \%$, and the fits for grain $\mathrm{T}_{111}$ are equally consistent.

\subsection{Material 2: rhombohedral fine-grained BNKT}

4.2.1. Sample preparation. Samples of $(0.82) \mathrm{Bi}_{0.5} \mathrm{Na}_{0.5^{-}}$ $\mathrm{TiO}_{3}-(0.18) \mathrm{Bi}_{0.5} \mathrm{~K}_{0.5} \mathrm{TiO}_{3}$ (BNKT) were produced by the mixed oxide route. Details of the synthesis method can be found elsewhere (Tran et al., 2011). The resulting grain size of the samples was approximately $3-5 \mu \mathrm{m}$. A sample was cut and polished into a rectangular shape of dimensions $100 \times 200 \times$ $90 \mu \mathrm{m}$. Gold electrodes were sputtered onto two opposing $100 \times 200 \mu \mathrm{m}$ surfaces.

4.2.2. Data acquisition. X-ray diffraction experiments were performed at beamline ID11 of the European Synchrotron Radiation Facility. A beam energy of $78.395 \mathrm{keV}$ (Pt $K$ edge) was focused and trimmed to a planar beam of dimensions $50 \mu \mathrm{m}$ in width $\times 5 \mu \mathrm{m}$ in height at the sample position. During the rotation around $\omega$ data were collected over $2 \times$ $160^{\circ}$ in $0.25^{\circ}$ integration angles with an exposure time of $5 \mathrm{~s}$ per image. A FReLoN detector (Labiche et al., 2007) with $2048 \times 2048$ pixels of $50 \times 50 \mu \mathrm{m}$ was employed at a sampleto-detector distance of $496 \mathrm{~mm}$. After the collection of an initial data set in the unpoled state, the sample was poled with an electric field of $4 \mathrm{kV} \mathrm{mm}^{-1}$ and a subsequent data set collected. Upon electrical poling the phase of the sample changed irreversibly from a pseudo-cubic to rhombohedral symmetry.

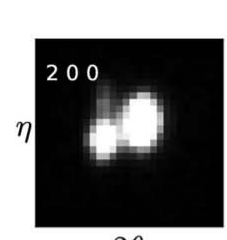

$2 \theta$

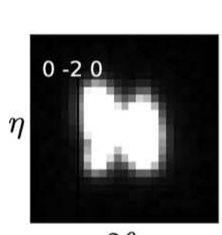

$2 \theta$

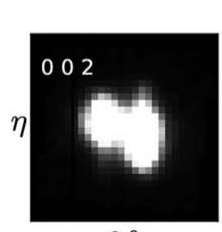

$2 \theta$
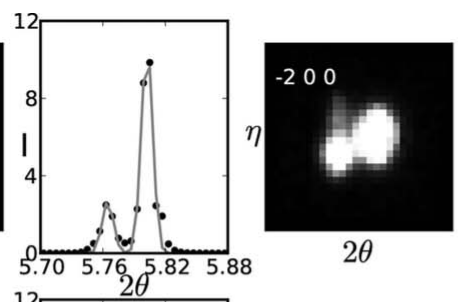

$2 \theta$

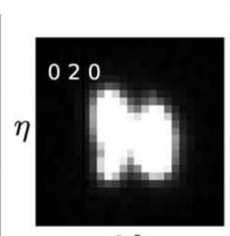

$2 \theta$

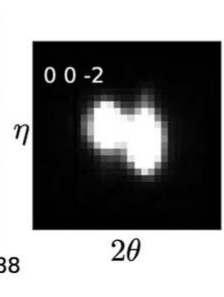

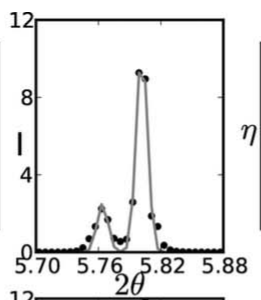

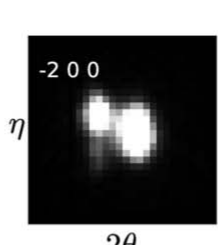

$2 \theta$
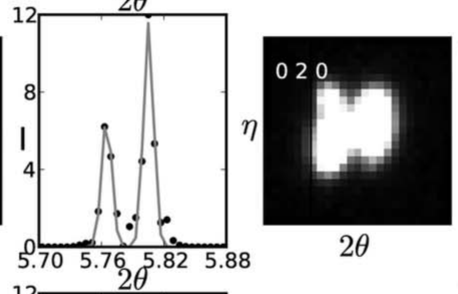

$2 \theta$

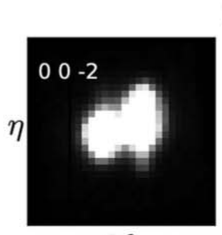

$2 \theta$
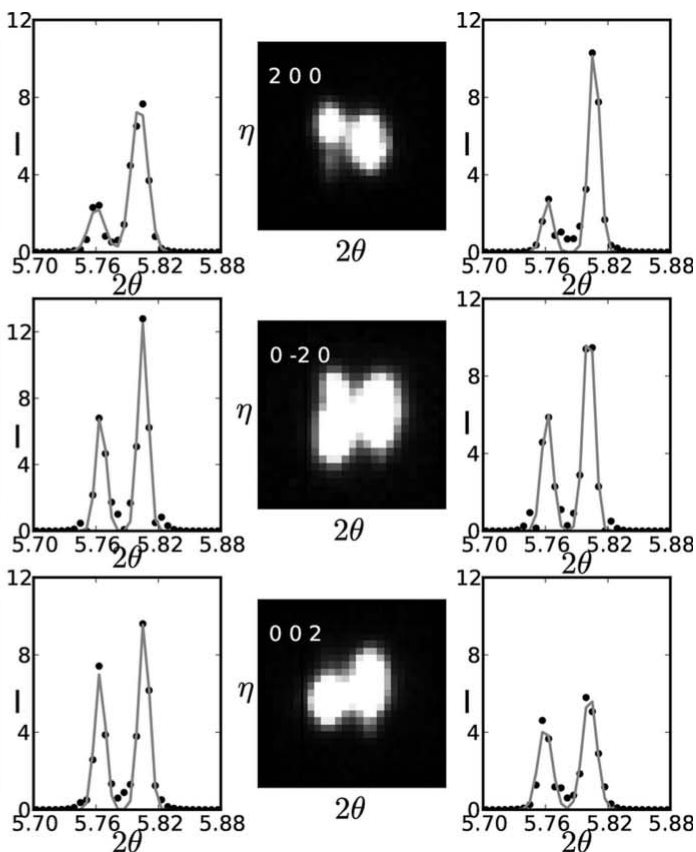

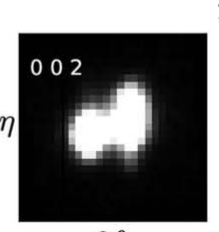

$2 \theta$

Figure 3

Reflections of the $\{002\}$ family for a tetragonal grain of BCZT, marked as $T_{100}$ in Table 1 . The intensity scale for the integrated data has a maximum of 12000 . The dots represent the measured data and the lines are the Gaussian peak fits. It can be seen that $v_{200}<v_{020}<v_{002}$ as anticipated, since $d_{002}$ is favourably aligned with its polarization axis approximately $7^{\circ}$ from the electric field axis. 
4.2.3. Grain indexing and selection. From the powder diffraction pattern of the same sample, the pseudo-cubic lattice parameter of BNKT in the unpoled state was refined to $a=3.905 \AA$, while the rhombohedral lattice parameters after poling were refined to $a=3.905 \AA$ and $\alpha_{\mathrm{rh}}=89.69^{\circ}$. The orientations of the grains in the poled state were determined using GrainSpotter (Schmidt, 2014). Since the sample cross section is larger than the beam width, grains will move in and out of the beam. By using a high completeness cutoff in terms of the number of observed to expected reflections, the indexing can be limited to the approximately 500 grains that are located within the central part of the sample, which is illuminated throughout the rotation. Here the completeness criterion was set to only include grains where all 16 possible $\{001\}$ and $\{002\}$ reflections were present, since these do not split in the $2 \theta$ dimension for rhombohedral symmetry. From the 500 central grains, two grains with the poling axis close to the crystallographic [100] $\left(R_{100}\right)$ and [111] $\left(R_{111}\right)$ axes were selected for further analysis.

4.2.4. Domain volume fitting. The selected grains $R_{100}$ and $R_{111}$ fulfil the criterion that reflections corresponding to all four domain variants were observed. This was deemed necessary here, as opposed to the coarse-grained BCZT case, in order to fit consistent domain volume fractions in the presence of significant peak overlap. In the domain volume fraction minimization [equation (2)] the last 111 and the last two $1 \overline{1} 1$ reflections shown in Fig. 4 were down-weighted owing to the intensity offsets caused by significant peak overlap. The weights ranged from 0.2 to 7.7 , an order of magnitude more than for BCZT, and they were only assigned on the basis of overall intensity consistency. A similar degree of inconsistency was observed for grain $R_{111}$. However, because of the volume weighting, the resulting absolute error bars on the poling strains of the rhombohedral grains $R_{100}$ and $R_{111}$ remain less than $0.03 \%$.

\subsection{Results}

Table 1 summarizes the domain volume fractions, poling strains and corresponding error estimates for the four selected grains, $T_{100}, T_{111}, R_{100}$ and $R_{111}$, described in the previous sections. Here $\alpha_{200}, \alpha_{020}$ and $\alpha_{002}$ are defined as the angles between the electric field vector and the $c$ axis of the tetragonal domains $d_{200}, d_{020}$ and $d_{002}$, respectively. Likewise, the angles $\alpha_{\overline{1} 11}, \alpha_{1 \overline{1} 1}, \alpha_{11 \overline{1}}$ and $\alpha_{111}$ are defined between the electric field direction and the body diagonals of the respective rhombohedral domains $d_{\overline{1} 11}, d_{1 \overline{1} 1}, d_{11 \overline{1}}$ and $d_{111}$. If only the domain variant with the smallest corresponding angle is populated, $\varepsilon_{\mathrm{p}}=\varepsilon_{\mathrm{p}}^{\mathrm{sat}}$ is obtained. For the tetragonal BCZT grains $T_{100}$ and $T_{111}$ the remnant poling strains are roughly an order of magnitude smaller than the theoretical saturated strains, and $\varepsilon_{\mathrm{p}}\left(T_{100}\right)>\varepsilon_{\mathrm{p}}\left(T_{111}\right)$ as anticipated from Fig. 2(a). The rhombohedral grains display the opposite trend in poling strain, $\varepsilon_{\mathrm{p}}\left(R_{100}\right)<\varepsilon_{\mathrm{p}}\left(R_{111}\right)$ [see Fig. 2(b)]. The overall remnant strain level in the rhombohedral BNKT sample is about half of the theoretical saturated strain level. Both this difference in strain level between the two samples and the orientation dependence of poling strains for the selected grains of the different symmetries are displayed in Fig. 5. The position of the grains in the stereographic triangle corresponds to the orientation relative to the electric field vector, and the colour code is the observed poling strain due to domain wall motion.

Considering the difference in internal consistency between reflections assigned to a grain of coarse-grained tetragonal BCZT (Fig. 3) and one of fine-grained rhombohedral BNKT
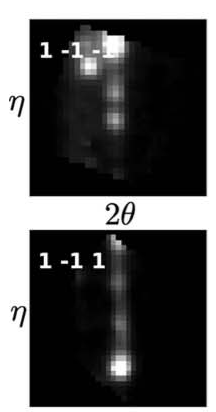

$2 \theta$

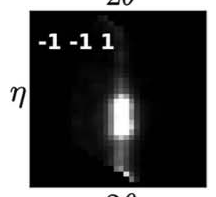

$2 \theta$

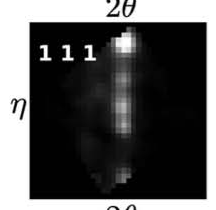

$2 \theta$
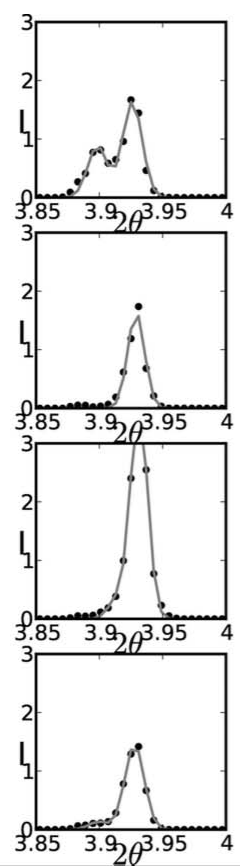
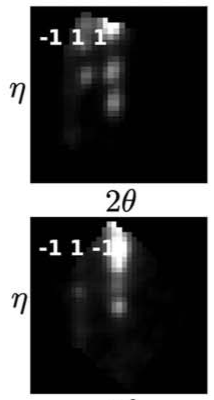

$2 \theta$
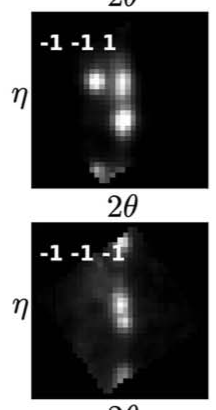

$2 \theta$
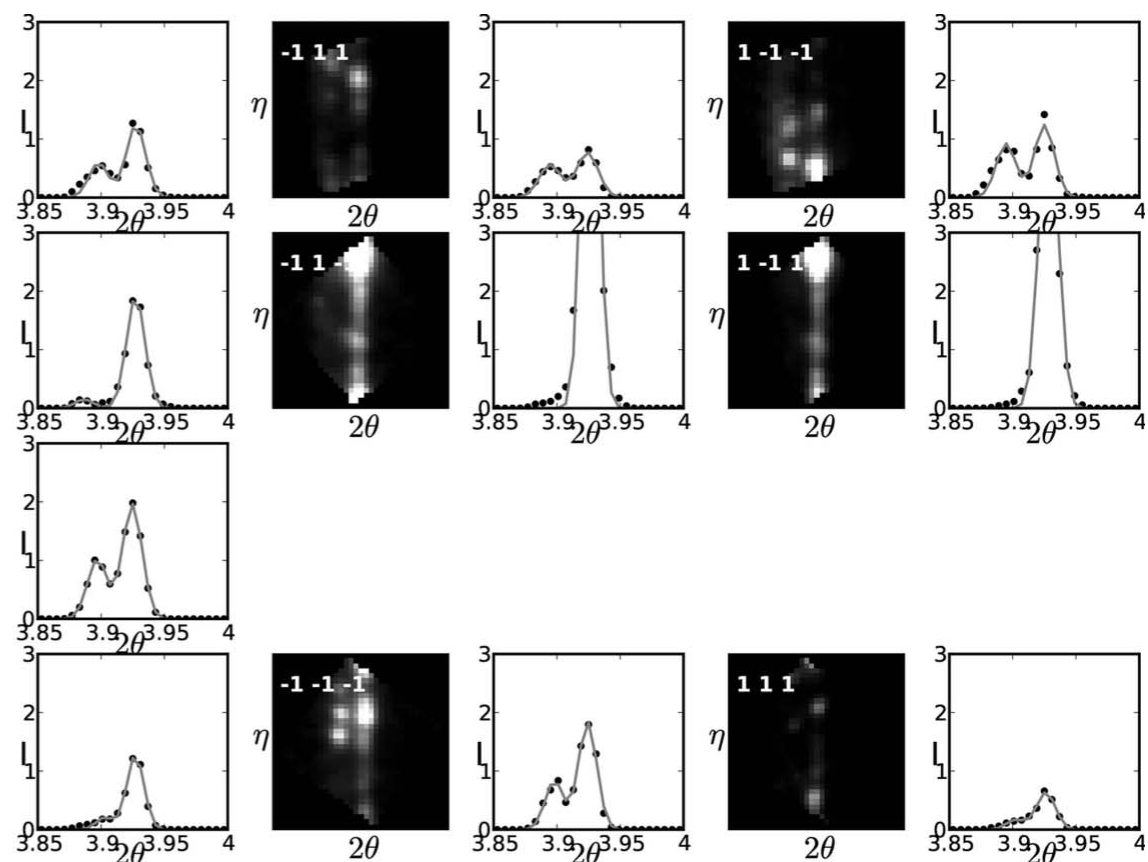
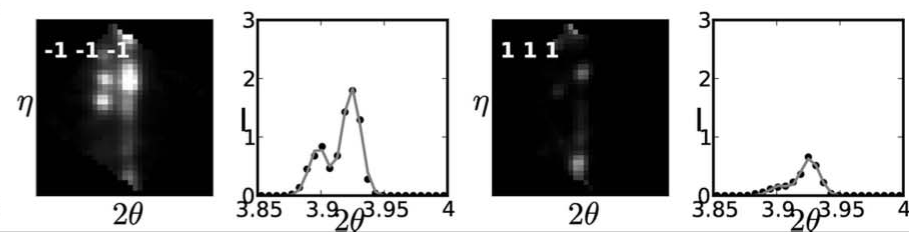

Figure 4

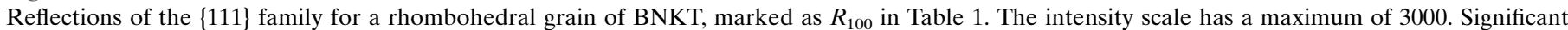
splitting is observed for all domain types except $d_{1 \overline{1} 1}$, consistent with a low volume fraction, $v_{1 \overline{1} 1}=0.07(2)$, as stated in Table 1 . 


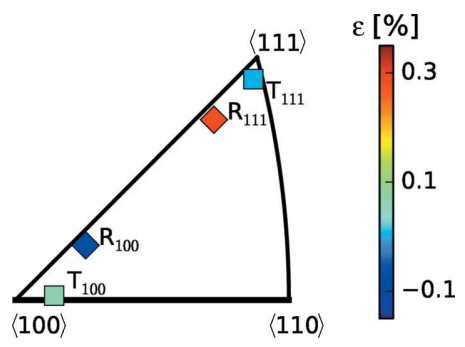

Figure 5

Orientations and poling strains for the four selected grains in Table 1. For the tetragonal grains the maximum poling strain is observed when the electric field vector is close to a $\langle 100\rangle$ axis, while the maximum poling strain for the rhombohedral grains is when the electric field direction is aligned close to a $\langle 111\rangle$ axis (see Fig. 2).

(Fig. 4), it is not surprising that the average absolute error bar on the poling strain for all grains, not just the ones selected in Table 1 , is approximately $0.01 \%$ for BCZT and $0.03 \%$ for BNKT. The reason for this is most likely the increased number of diffraction spots in the fine-grained BNKT compared to coarse-grained BCZT, which increases the likelihood of peak overlap and hence erroneous peak intensities. However, as noted above, the remnant strains observed in the rhombohedral BNKT are also substantially larger than in the tetragonal $\mathrm{BCZT}$, so the relative errors bars in each case are comparable.

\section{Discussion}

The measurement method demonstrated gives unique access to grain-scale domain volume fraction and resultant non- $180^{\circ}$ ferroelectric domain switching strains. This information can be used to advance our understanding of ferroic materials at the grain scale and will be critical for experimental verification of mesoscopic materials modelling efforts, for instance phase field models, which have increasing relevance to the study of ferroic systems (Potnis et al., 2011).

The examples in $\$ 4$ demonstrate that peak overlap is the main limitation of the present algorithm for fitting individual domain volume fractions. In order to avoid possible experimental artefacts from the use of this method, the following factors should be considered when measuring from coarseand fine-grained materials:

(1) Grain sizes $>10 \mu \mathrm{m}$

(a) Use a beam height that is roughly double the average grain size and consider at least five non-overlapping layers to get sufficient statistics from fully embedded grains.

(2) Grains sizes $<10 \mu \mathrm{m}$

(a) Use a sample with a smaller cross section, ideally smaller than the beam width, though this presents its own experimental difficulties, particularly for in situ measurements owing to processing difficulties.

(b) Scanning beam methods (Bonnin et al., 2014) may be employed in order to also overcome the total number of grains in the beam for any particular orientation.

Further advances of the method can be envisioned. Three significant pieces of information are missing from such an analysis: (1) the spatial distribution of domains within the grain, (2) the orientation relationships between domains within grains and (3) the piezoelectric or elastic compliance strain response of the individual domain variants. It is possible that the spatial distribution of domains could be extracted from the current method if it is combined with space-filling grain maps measured using a high-resolution near-field detector (Schmidt et al., 2008; Ludwig et al., 2008, 2009; Li et al., 2012; Reischig et al., 2013; Pokharel et al., 2014). However, here the minimum domain size would probably have to be of the order of $10 \mu \mathrm{m}$ in order to resolve such structures. Additional X-ray microscopy methods are being developed, which allow for much higher spatial resolution of sub-grain structures in three dimensions from bulk materials (Simons et al., 2015). It is envisioned that such methods may progress towards $50 \mathrm{~nm}$ resolution with improved source and optics characteristics, opening up many opportunities for the study of domain structures and their influence on properties in bulk polycrystalline materials.

The orientation relationships between domains within a grain may also be obtained from the far-field 3D-XRD data (Varlioglu et al., 2010), as the domains are simply entities with distinct orientations and can be indexed in the same way as twins in metallic materials (Aydıner et al., 2009; Oddershede et al., 2011; Bieler et al., 2014; Abdolvand et al., 2015). However, the diffraction spots arising from ferroelastic domains of the same grain in electroceramic materials are not nearly as well separated in $2 \theta, \eta$ and $\omega$ as the diffraction spots of parent and twin pairs in previously measured metallic materials, and many reflections are in fact shared between two or more domains.

In addition to the non- $180^{\circ}$ domain switching strains calculated here, the system will also undergo elastic strains due to the intrinsic piezoelectric effect and compliance strains due to the deformation of surrounding grains. Observing these elastic strain responses of the individual domain variants within the measured grains could also potentially be done if the above two extensions could be achieved. This would allow unprecedented information on the response of ferroic materials in the bulk.

The mathematical formulation and application examples presented here have focused on tetragonal and rhombohedral crystallographic symmetry. However, the algorithm for determining domain volume fractions can be extended to work for orthorhombic domain structures by investigating the threefold splitting in any pseudocubic family of reflections $\{h k l\}$ except those fulfilling $|h|=|k|=|l|$, or for lower symmetries by using combinations of reflection sets. Similarly, extensions to account for domain volume fraction changes in mixed phase systems could be achieved by considering multiple reflection families.

\section{Conclusions and outlook}

A method has been developed to allow for the measurement of domain volume fractions and associated domain switching strains from isolated grains within a bulk polycrystalline material. The method was demonstrated on two contrasting materials, namely, a coarse-grained (approximately $50 \mu \mathrm{m}$ ) 
BCZT and a fine-grained (approximately $3 \mu \mathrm{m}$ ) BNKT. In these cases, absolute domain switching strain errors of 0.01 and $0.03 \%$ were found, respectively. It is hoped that the currently presented method will be extended in the future to allow studies incorporating not only domain volume fractions but also spatial distributions, orientation distributions and elastic deformation of individual ferroelastic domains. The methods presented are extendable to multiple ferroic material types. They have the potential to enhance our understanding of ferroic behaviour in the polycrystalline state and will provide critical mesoscale information for the confirmation and/or constraint of modelling efforts at these length scales.

\section{Acknowledgements}

The authors acknowledge support from the Danish Independent Research Council | Technology and Production Sciences case No. 12-127449 and Australian Research Council Discovery Project DP120103968. The Danish Independent Research Council | Natural Sciences is acknowledged for covering expenses in relation to the synchrotron experiment (through Danscatt). This research used resources of the Advanced Photon Source, a US Department of Energy (DOE) Office of Science User Facility operated for the DOE Office of Science by Argonne National Laboratory under contract No. DE-AC02-06CH11357. The European Synchrotron Radiation Facility (MA-1919) and Advanced Photon Source (GUP-32411) are acknowledged for granting beamtime for the experiments. JED acknowledges support from an Australian Institute of Nuclear Science and Engineering research fellowship. Finally the authors wish to thank Julia Glaum, UNSW Australia, and Wook Jo, UNIST, South Korea, for providing the sample materials for the current study.

\section{References}

Abdolvand, H., Majkut, M., Oddershede, J., Schmidt, S., Lienert, U., Diak, B. J., Withers, P. J. \& Daymond, M. R. (2015). Int. J. Plasticity, 70, 77-97.

Arlt, G. \& Sasko, P. (1980). J. Appl. Phys. 51, 4956-4960.

Aydıner, C. C., Bernier, J. V., Clausen, B., Lienert, U., Tomé, C. N. \& Brown, D. W. (2009). Phys. Rev. B, 80, 024113.

Azimonte, C., Granado, E., Terashita, H., Park, S. \& Cheong, S. W. (2010). Phys. Rev. B, 81, 012103.

Bernier, J. V., Barton, N. R., Lienert, U. \& Miller, M. P. (2011). J. Strain Anal. Eng. Des. 46, 527-547.

Bieler, T. R., Wang, L., Beaudoin, A. J., Kenesei, P. \& Lienert, U. (2014). Met. Mater. Trans. A, 45, 109-122.

Bonnin, A., Wright, J. P., Tucoulou, R. \& Palancher, H. (2014). Appl. Phys. Lett. 105, 084103.

Daniels, J. E., Pramanick, A. \& Jones, J. L. (2009). IEEE Trans. Ultrason. Ferroelectr. Freq. Control, 56, 1539-1545.

Gruverman, A., Kolosov, O., Hatano, J., Takahashi, K. \& Tokumoto, H. (1995). J. Vac. Sci. Technol. B, 13, 1095-1099.

Hall, D. A., Steuwer, A., Cherdhirunkorn, B., Mori, T. \& Withers, P. J. (2004). J. Appl. Phys. 96, 4245-4252.

Hall, D. A., Steuwer, A., Cherdhirunkorn, B., Mori, T. \& Withers, P. J. (2006). Acta Mater. 54, 3075-3083.
Hall, D. A., Steuwer, A., Cherdhirunkorn, B., Withers, P. J. \& Mori, T. (2005). Mater. Sci. Eng. A, 409, 206-210.

Harrison, R. J. (2004). J. Appl. Phys. 95, 1706-1717.

Juul Jensen, D., Lauridsen, E. M., Margulies, L., Poulsen, H. F., Schmidt, S., Sørensen, H. O. \& Vaughan, G. M. B. (2006). Mater. Today, 9, 18-25.

Labiche, J.-C., Mathon, O., Pascarelli, S., Newton, M. A., Ferre, G. G., Curfs, C., Vaughan, G., Homs, A. \& Carreiras, D. F. (2007). Rev. Sci. Instrum. 78, 091301.

Levenberg, K. (1944). Q. Appl. Math. 2, 164-168.

Li, S. F., Lind, J., Hefferan, C. M., Pokharel, R., Lienert, U., Rollett, A. D. \& Suter, R. M. (2012). J. Appl. Cryst. 45, 1098-1108.

Li, W., Xu, Z., Chu, R., Fu, P. \& Zang, G. (2010). Mater. Lett. 64, 23252327.

Liu, W. \& Ren, X. (2009). Phys. Rev. Lett. 103, 257602.

Ludwig, W., King, A., Reischig, P., Herbig, M., Lauridsen, E. M., Schmidt, S., Proudhon, H., Forest, S., Cloetens, P., du Roscoat, S. R., Buffière, J., Marrow, T. \& Poulsen, H. (2009). Mater. Sci. Eng. A, 524, 69-76.

Ludwig, W., Schmidt, S., Lauridsen, E. M. \& Poulsen, H. F. (2008). J. Appl. Cryst. 41, 302-309.

Lupascu, D. C., Hoffmann, M. J., Hammer, M. \& Endriss, A. (2001). Acta Mater. 49, 1301-1310.

Marquardt, D. (1963). J. Soc. Ind. Appl. Math. 11, 431-441.

Oddershede, J., Schmidt, S., Poulsen, H. F., Margulies, M., Wright, J., Moscicki, M., Reimers, W. \& Winther, G. (2011). Mater. Charact. 62, 651-660.

Oddershede, J., Schmidt, S., Poulsen, H. F., Sørensen, H. O., Wright, J. \& Reimers, W. (2010). J. Appl. Cryst. 43, 539-549.

Pokharel, R., Lind, J., Kanjarla, A., Lebensohn, R., Li, S. F., Kenesei, P., Suter, R. M. \& Rollett, A. D. (2014). Annu. Rev. Condens. Matter Phys. 5, 317-346.

Potnis, P. R., Tsou, N.-T. \& Huber, J. E. (2011). Materials, 4, 417-447. Poulsen, H. F. (2004). Three-Dimensional X-ray Diffraction Microscopy. Mapping Polycrystals and Their Dynamics, Tracts in Modern Physics, Vol. 205. Berlin: Springer-Verlag.

Poulsen, H. F., Nielsen, S. F., Lauridsen, E. M., Schmidt, S., Suter, R. M., Lienert, U., Margulies, L., Lorentzen, T. \& Juul Jensen, D. (2001). J. Appl. Cryst. 34, 751-756.

Pramanick, A., Damjanovic, D., Daniels, J. E., Nino, J. C. \& Jones, J. L. (2011). J. Am. Ceram. Soc. Feature Art. 94, 293-309.

Reischig, P., King, A., Nervo, L., Viganó, N., Guilhem, Y., Palenstijn, W. J., Batenburg, K. J., Preuss, M. \& Ludwig, W. (2013). J. Appl. Cryst. 46, 297-311.

Schmidt, S. (2014). J. Appl. Cryst. 47, 276-284.

Schmidt, S., Olsen, U. L., Poulsen, H. F., Sørensen, H. O., Lauridsen, E. M., Margulies, L., Maurice, C. \& Juul Jensen, D. (2008). Scr. Mater. 59, 491-494.

Seidel, J. et al. (2009). Nat. Mater. 8, 229-234.

Simons, H., King, A., Ludwig, W., Detlefs, C., Pantleon, W., Schmidt, S., Snigireva, I., Snigirev, A. \& Poulsen, H. F. (2015). Nat. Commun. 6, 6098 .

Sørensen, H. O., Schmidt, S., Wright, J. P., Vaughan, G. B. M., Techert, S., Garman, E. F., Oddershede, J., Davaasambuu, J., Paithankar, K. S., Gundlach, C. \& Poulsen, H. F. (2012). Z. Kristallogr. 227, 63-78. Tan, X. L., He, H. \& Shang, J. K. (2005). J. Mater. Res. 20, 1641-1653. Tran, V. D. N., Han, H.-S., Yoon, C.-H., Lee, J.-S., Jo, W. \& Rödel, J. (2011). Mater. Lett. 65, 2607-2609.

Varlioglu, M., Lienert, U., Park, J.-S., Jones, J. L. \& Üstündag, E. (2010). Texture Stress Microstruct. 2010, 910793.

Wang, Z., Webber, K. G., Hudspeth, J. M., Hinterstein, M. \& Daniels, J. E. (2014). Appl. Phys. Lett. 105, 161903.

Wu, H., Li, L., Liang, L.-Z., Liang, S., Zhu, Y.-Y. \& Zhu, X.-H. (2015). J. Eur. Ceram. Soc. 35, 411-441.

Zhi, Y., Liu, D., Sun, J., Yan, A., Zhou, Y., Luan, Z., Dai, E., Liu, L. \& Qu, W. (2009). J. Appl. Phys. 105, 024106. 